\title{
Aberrant Expression of Pseudogene- Derived IncRNAs as an Alternative Mechanism of Cancer Gene Regulation in Lung Adenocarcinoma
}

\author{
Greg L. Stewart ${ }^{1 *}$, Katey S. S. Enfield ${ }^{1,2}$, Adam P. Sage $^{1}$, Victor D. Martinez ${ }^{1}$, \\ Brenda C. Minatel ${ }^{1}$, Michelle E. Pewarchuk ${ }^{1}$, Erin A. Marshall ${ }^{1}$ and Wan L. Lam ${ }^{1}$
}

${ }^{1}$ BC Cancer Research Centre, Vancouver, BC, Canada, ${ }^{2}$ The Francis Crick Institute, London, United Kingdom

\section{OPEN ACCESS}

Edited by:

Yujing Li,

Emory University, United States

Reviewed by:

Sergio Verjovski-Almeida,

University of São Paulo, Brazil

Nithyananda Thorenoor,

Pennsylvania State University,

United States

*Correspondence:

Greg L. Stewart

gstewart@bccrc.ca

Specialty section:

This article was submitted to

$R N A$,

a section of the journal

Frontiers in Genetics

Received: 16 November 2018

Accepted: 11 February 2019

Published: 06 March 2019

Citation:

Stewart GL, Enfield KSS, Sage AP, Martinez VD, Minatel BC,

Pewarchuk ME, Marshall EA and Lam WL (2019) Aberrant Expression of Pseudogene- Derived IncRNAs as an Alternative Mechanism of Cancer

Gene Regulation in Lung Adenocarcinoma.

Front. Genet. 10:138

doi: 10.3389/fgene.2019.00138
Transcriptome sequencing has led to the widespread identification of long non-coding RNAs (IncRNAs). Subsequently, these genes have been shown to hold functional importance in human cellular biology, which can be exploited by tumors to drive the hallmarks of cancer. Due to the complex tertiary structure and unknown binding motifs of IncRNAs, there is a growing disparity between the number of IncRNAs identified and those that have been functionally characterized. As such, IncRNAs deregulated in cancer may represent critical components of cancer pathways that could serve as novel therapeutic intervention points. Pseudogenes are non-coding DNA sequences that are defunct relatives of their protein-coding parent genes but retain high sequence similarity. Interestingly, certain IncRNAs expressed from pseudogene loci have been shown to regulate the protein-coding parent genes of these pseudogenes in trans particularly because of this sequence complementarity. We hypothesize that this phenomenon occurs more broadly than previously realized, and that aberrant expression of IncRNAs overlapping pseudogene loci provides an alternative mechanism of cancer gene deregulation. Using RNA-sequencing data from two cohorts of lung adenocarcinoma, each paired with patient-matched non-malignant lung samples, we discovered 104 deregulated pseudogene-derived IncRNAs. Remarkably, many of these deregulated IncRNAs (i) were expressed from the loci of pseudogenes related to known cancer genes, (ii) had expression that significantly correlated with protein-coding parent gene expression, and (iii) had IncRNA protein-coding parent gene expression that was significantly associated with survival. Here, we uncover evidence to suggest the IncRNApseudogene-protein-coding gene axis as a prominent mechanism of cancer gene regulation in lung adenocarcinoma, and highlights the clinical utility of exploring the non-coding regions of the cancer transcriptome.

Keywords: long non-coding RNAs, pseudogenes, lung cancer, gene regulation, non-coding RNAs 


\section{INTRODUCTION}

Lung cancer is an enormous health burden, representing the most common cause of cancer death worldwide. While advances in imaging technology have improved early detection, the outcome for lung cancer patients remains poor. This is largely attributed to limited therapeutic options and short-term response due to tumor heterogeneity. Despite extensive sequencing efforts to characterize exomes and protein-coding transcriptomes of lung tumors, less than half of lung adenocarcinoma (LUAD) patients harbor clinically targetable mutated driver genes, highlighting the need to explore alternative mechanisms of cancer gene deregulation (Chan and Hughes, 2015).

Long non-coding RNAs (lncRNAs, > $200 \mathrm{nt}$ ) have emerged as important players in cell biology. LncRNAs have been observed to function through a wide variety of regulatory mechanisms, targeting DNA, proteins, and other RNA species; specifically with defined roles in RNA degradation or stabilization, protein translocation and complex formation, and recruitment of complexes to transcriptional loci (Lee et al., 1999; Wang et al., 2011; Kung et al., 2013; Simon et al., 2013). This broad functional repertoire has been shown to be exploited by many cancer types, including LUAD, to drive various hallmarks of cancer (Bhan et al., 2017; Peng et al., 2017). One of the first cancerassociated lncRNA transcripts, MALAT1 (Metastasis Associated Lung Adenocarcinoma Transcript 1) was discovered in lung cancer. MALAT1 is overexpressed in metastatic lung tumors and functions in trans through transcriptional regulation of multiple genes involved in cell motility (Tano et al., 2010; Gutschner et al., 2013). Since the discovery of MALAT1, many other lncRNAs have been shown to play a direct role in nearly every major cancer type. Tumor suppressive lncRNA transcripts are commonly observed to be downregulated, for instance the lncRNA TARID, which promotes the expression of the tumor suppressor gene TCF21 through active promoter demethylation (Arab et al., 2014). Conversely oncogenic lncRNAs can be overexpressed, such as NEAT1, an architectural lncRNA associated with metastasis in many cancer types (Hirose et al., 2014; Blume et al., 2015; Yamazaki et al., 2018; Zhang et al., 2018). As such, lncRNAs may represent critical regulators of oncogenic-driver pathways that could serve as undiscovered clinical intervention points in LUAD.

While lncRNAs have been observed to be important in cancer biology, functional prediction of newly-discovered lncRNAs remains a major challenge. However, many lncRNAs expressed from pseudogene loci have been shown to regulate the specific genes with which they have sequence homology. Pseudogenes are DNA sequences that are defunct relatives of functional proteincoding genes (herein referred to as parent genes) and arise during either gene duplication events, or the reverse transcription of an mRNA transcript into a new genomic location. Through evolution these duplicated genes have acquired mutations such as premature stop codons and frameshifts, which results in the loss of protein coding ability, while still retaining a high degree of sequence homology with the original parent gene (Khachane and Harrison, 2009). Recently, pseudogene-derived lncRNAs have been shown to regulate their parent genes and this novel mechanism has been observed in many tumor types, including lung cancer (Sun et al., 2017; Hu et al., 2018). A prominent example is the tumor suppressor gene PTEN (chromosome 10), regulated both positively by PTENP1 (chromosome 9), a lncRNA transcribed from the sense strand of the pseudogene locus, and negatively by the IncRNA PTENP1-AS1, which is transcribed from the strand antisense to the parent gene (Johnsson et al., 2013).

Pseudogenes have been continually omitted from large RNAsequencing datasets due to the complexity of separating highly similar pseudogene sequences from parent genes. However, Milligan et al. (2016) recently generated an atlas of lncRNAs overlapping pseudogenes, which has provided a foundation for their analysis in RNA sequencing datasets. We hypothesize that the functions of pseudogene-derived lncRNAs are an underexplored mechanism of gene regulation that occurs more broadly than previously realized, and that these events contribute to the tumorigenesis of LUAD. We performed next generation RNAsequencing on microdissected LUAD tumors and matched nonmalignant tissue to identify deregulated lncRNAs expressed from pseudogene loci (herein referred to as $\Psi$-lncs). We then explored the relationships of these $\Psi$-lncs with their parent genes, and explored their significance in relation to patient clinical features in our discovery dataset as well as a validation dataset.

\section{RESULTS}

\section{$\Psi$-Inc Expression Is Deregulated in Lung Adenocarcinoma}

Pseudogenes vary widely in terms of length, gene fraction, and identity to parent genes, and can be expressed as lncRNAs that are sense, antisense, partial overlapping, or internal to the parent. In light of this variation, $\Psi$-lncs are observed to have vastly different regulatory effects on downstream target genes (Figure 1A). In our curation of $\Psi$-lncs in LUAD, we have included those that have exonic overlap with a pseudogene (partial or full length) and considered both sense and antisense transcripts (Supplementary Table S1). $\Psi$-lncs were analyzed in an in-house discovery (BCCA, $n=72$ ) and external validation (TCGA, $n=108$ ) cohort of LUAD and paired non-malignant lung tissues (Table 1). We identified aberrantly expressed $\Psi$-lncs that are significantly deregulated in both the discovery and validation datasets with the same direction of expression alteration ( $\Psi$-lncs upregulated or downregulated in tumors compared to matched non-malignant tissue).

We found 104 lncRNAs expressed from 102 pseudogene loci to be significantly deregulated in LUAD (Supplementary Table S2). To our surprise, we found that the majority of these deregulated $\Psi$-lncs were downregulated in tumors (Figures 2A,B). Most of these were unannotated lncRNAs, such as RP11-1007O24.3, which was downregulated in tumors, with only 24 of the total deregulated $\Psi$-lncs having been previously described in scientific literature annotated in PubMed, albeit none in the field of pseudogene-mediated deregulation (Supplementary Table S3 and Figure 2B). Twenty of these 24 have been described in the context of cancer, with only four in lung cancer. This includes DGCR5, a lncRNA we found to be overexpressed in 
A

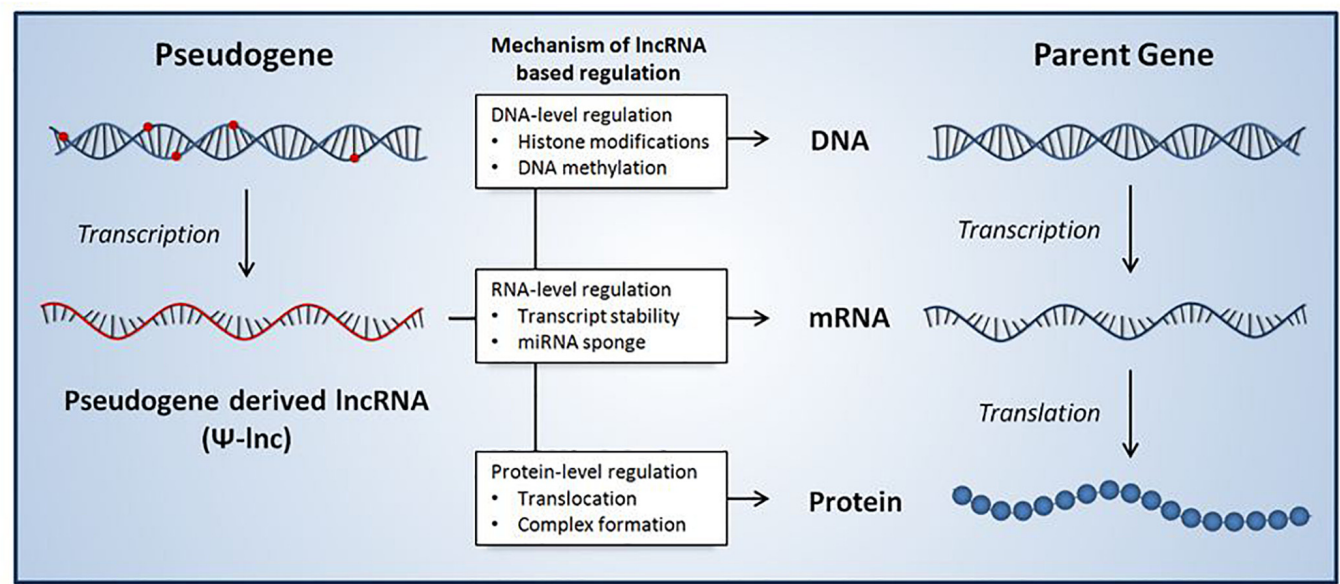

B

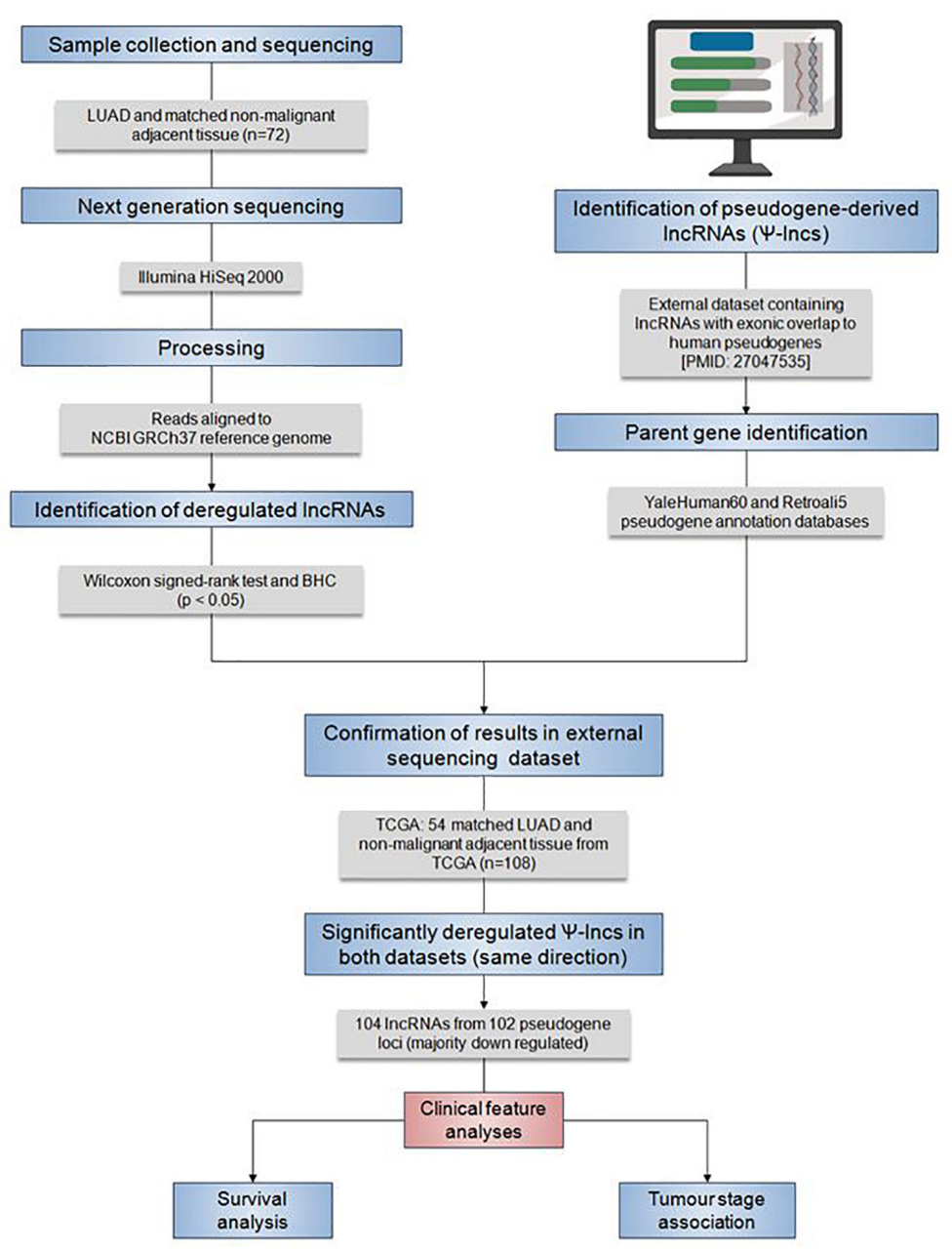

FIGURE 1 | Summary of the regulatory mechanisms of $\Psi$-Incs and the analysis pipeline for the identification of their deregulation in lung adenocarcinoma. (A) Summary of the regulatory mechanisms of pseudogene-derived IncRNAs ( $\Psi$-Incs) that retain sequence homology with the parent gene. Overall, IncRNAs have been shown to function through a variety of regulatory mechanisms, acting on the DNA, RNA, and protein levels. (B) Flow diagram description of the analysis pipeline applied for the identification of deregulated $\Psi$-Incs. Patient LUAD samples were collected and subjected to next generation sequencing to quantify RNA expression. Gene expression was then compared between tumors and matched non-malignant tissue to identify significantly deregulated transcripts. LncRNAs with exonic overlap to known pseudogenes were then identified and confirmed in a 2nd set of LUAD and matched non-malignant tissue. Deregulated $\Psi$-Incs were then assayed to determine associations with clinical features. 
TABLE 1 | Patient clinical characteristics of the discovery (BCCA), validation (TCGA), and survival analysis (KmPlotter) datasets.

\begin{tabular}{|c|c|c|c|}
\hline Characteristic & BCCA & TCGA & KmPlotter \\
\hline Samples (pairs) & $72(36)$ & $108(54)$ & 866 (n/a) \\
\hline \multicolumn{4}{|l|}{ Sex } \\
\hline Male & 10 & 24 & 344 \\
\hline Female & 26 & 30 & 318 \\
\hline Average age & 70 & 67 & - \\
\hline \multicolumn{4}{|l|}{ Stage } \\
\hline 1 & $20(56 \%)$ & $28(52 \%)$ & 370 (69\%) \\
\hline$\|$ & $11(31 \%)$ & $14(30 \%)$ & $136(25 \%)$ \\
\hline III & 3 (8\%) & 10 (19\%) & $24(4 \%)$ \\
\hline IV & $1(3 \%)$ & $2(4 \%)$ & $4(1 \%)$ \\
\hline \multicolumn{4}{|l|}{ Ethnicity } \\
\hline Caucasian & $11(31 \%)$ & $51(94 \%)$ & - \\
\hline Asian & 14 (39\%) & - & - \\
\hline Unknown & 11 (31\%) & - & - \\
\hline Black & - & $3(6 \%)$ & - \\
\hline \multicolumn{4}{|l|}{ Smoking } \\
\hline Current & $5(14 \%)$ & $7(13 \%)$ & 246 (Ever $^{a}$ \\
\hline Former & $6(17 \%)$ & $36(67 \%)$ & \\
\hline Never & 25 (69\%) & 5 (9\%) & 246 \\
\hline
\end{tabular}

a Ever smokers is a term that includes both current and former smokers.

tumors. DGCR5 has been reported to promote LUAD progression by sequestering a variety of miRNAs involved in cell cycle regulation, although it has not been investigated with regard to its pseudogene-derived nature (Chen et al., 2017; Dong et al., 2018; Luo et al., 2018).
We were interested in examining the genetic events that could impact pseudogene loci, and thus affect $\Psi$-lnc expression. We mapped the chromosomal distribution of the deregulated $\Psi$-lncs, finding them to be distributed throughout the genome and detected on most chromosomes, except for chromosomes 4 and Y (Figure 3). The locations of each of the parent genes of deregulated $\Psi$-lncs are similarly distributed through the genome (Supplementary Table S4). We then determined the overlap of these genes with regions of recurrent chromosomal amplification and deletion as determined by The Cancer Genome Atlas (TCGA) for LUAD (Zack et al., 2013). While some $\Psi$-lncs overlap with regions of recurrent deletion, the majority do not, indicating that they may be regulated by mechanisms other than copy number alteration (Figure 3).

\section{Global Patterns of $\Psi$-Inc and Parental Gene Expression}

As a first step to identify deregulated $\Psi$-lncs that may function through regulation of their respective parent genes, we explored whether $\Psi$-lncs with significantly deregulated expression were associated with altered parent gene transcript levels (Poliseno et al., 2010; Johnsson et al., 2013; Feng et al., 2017; Huang et al., 2018). We obtained parent gene information for the 95 deregulated $\Psi$-lncs and determined that they shared 104 parent genes. Some pseudogenes contained multiple lncRNAs, and some lncRNAs overlapped multiple pseudogenes, constituting a total number of $116 \Psi$-lnc-parent gene pairings. For each $\Psi$-lncparent pair we compared groups of tumors with high levels of $\Psi$-lnc expression to those with low levels of $\Psi$-lnc expression.
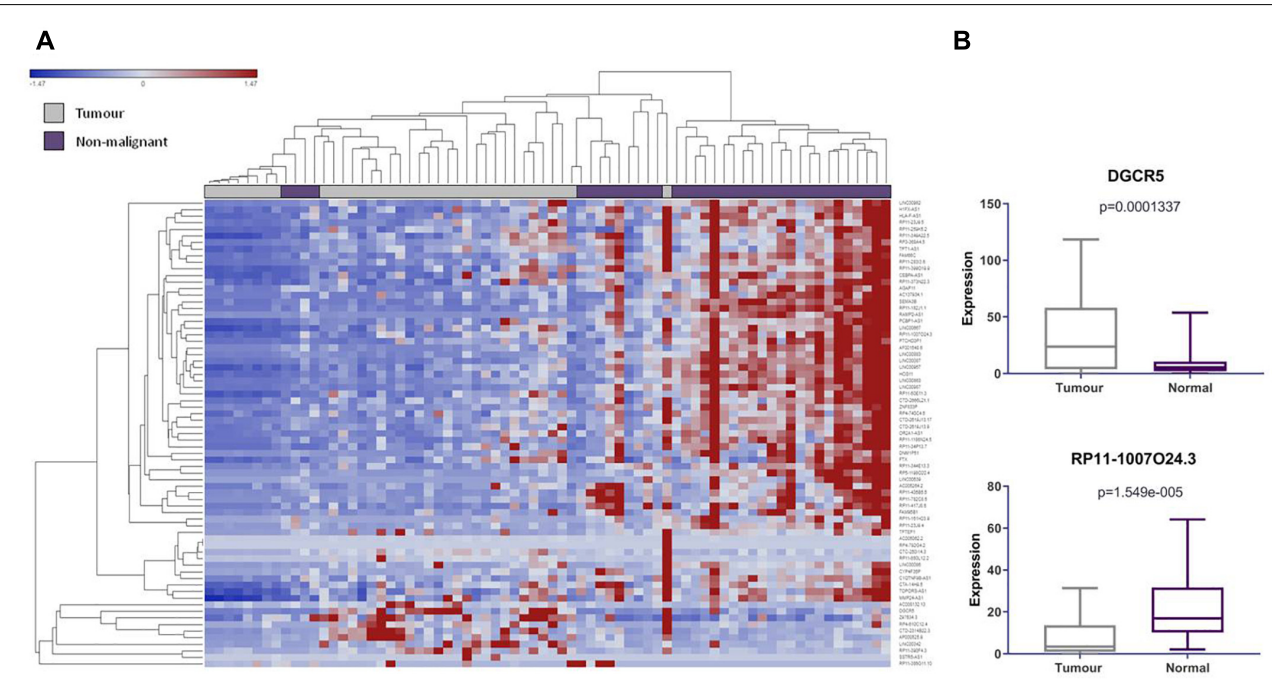

FIGURE 2 | LncRNAs derived from pseudogene loci are significantly differentially expressed in lung adenocarcinoma compared to matched non-malignant lung tissue. (A) Unsupervised hierarchical clustering of pseudogene-derived IncRNAs differentially expressed between lung adenocarcinoma (gray) and matched non-malignant tissue (purple). Average linkage was used as the cluster distance metric and Pearson Correlation was used as the point correlation metric. Expression values are stratified from low (blue) to high (red). Only pseudogene-derived IncRNAs with average expression values of greater than or equal to 10 RPKM were included in the clustering analysis. Clustering of samples highlights relative similarity in pseudogene-derived IncRNA expression between the two sample groups, while clustering of gene expression reveals a trend toward the widespread underexpression of these transcripts in lung adenocarcinoma. (B) Highlighted examples of pseudogene-derived IncRNAs significantly deregulated between lung tumors and non-malignant tissues. Expression (RPKM) in tumors (gray) and normal tissues (purple) is represented on the $\mathrm{Y}$-axis. Boxes represent the interquartile range and inner lines represent the median expression value. 


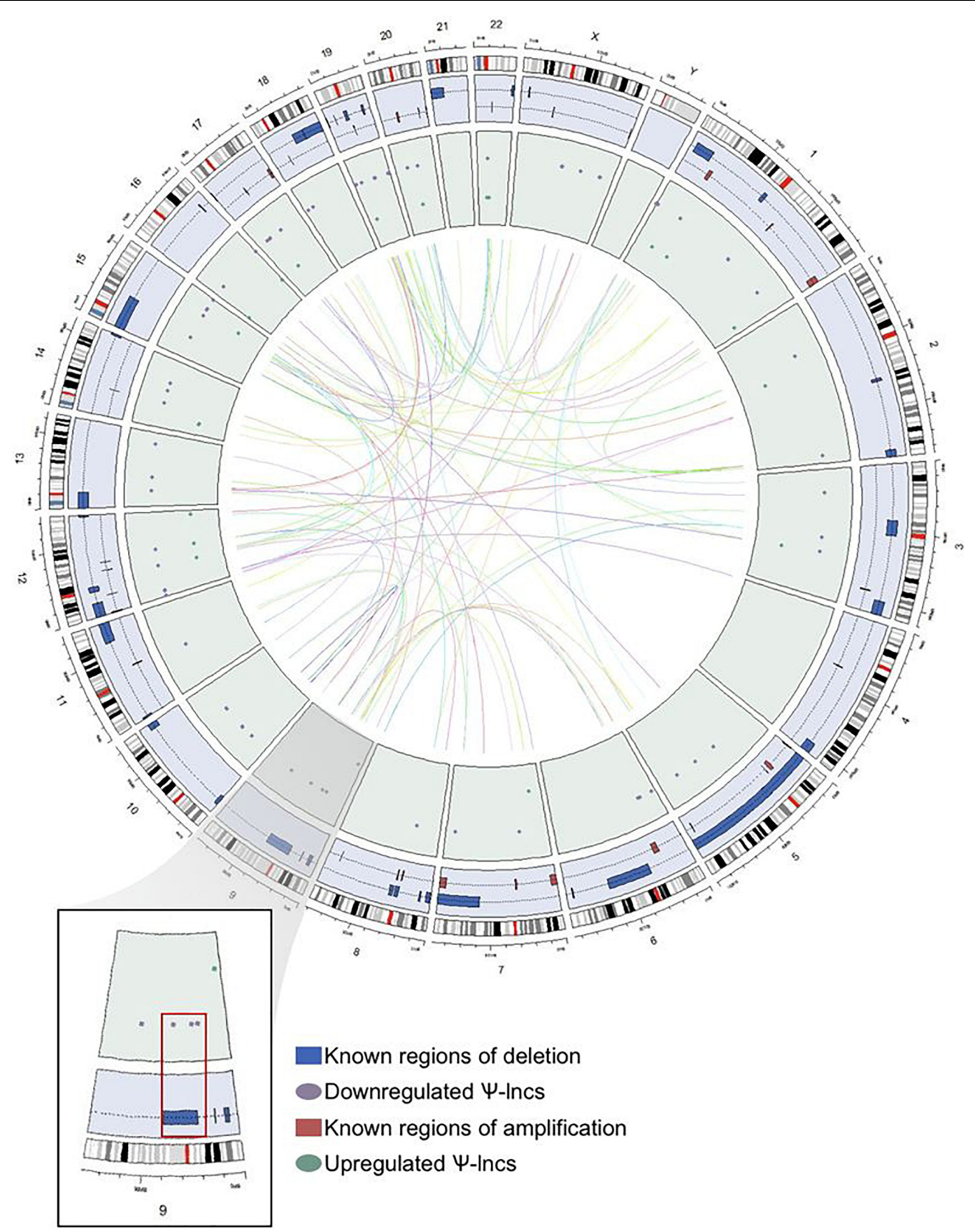

FIGURE 3 | Genome-wide distribution of deregulated pseudogene-derived IncRNAs in lung adenocarcinoma. Circular representation of the genomic distribution of the deregulated pseudogene-derived IncRNAs discovered in our study, as well as known regions of copy number alterations in lung adenocarcinoma as described by TCGA. The outer concentric circle represents the human karyotype from the genomic build hg19. The blue concentric circle contains known regions of copy number amplification (red boxes) and deletion (blue boxes) that have been previously published. The inner green circle represents the specific genomic location of our pseudogene-derived IncRNAs found to be either upregulated (green circles) or downregulated (purple circles) in lung adenocarcinoma. Finally, the inner connecting lines represent the interaction between the deregulated pseudogene-derived IncRNAs and the locations of their respective protein-coding parent genes. Chromosome 9 (magnified region) highlights that some of the downregulated pseudogene-derived IncRNAs overlap with genomic regions frequently deleted in lung adenocarcinoma.

We found that $33 \Psi$-lncs have a significant expression relationship with their parent gene in at least 1 dataset (Supplementary Table S5). This included 21 sense $\Psi$-lnc-parent-gene pairs, and 13 antisense $\Psi$-lnc-parent gene pairs. Having identified
$\Psi$-lncs with expression associated with parent gene expression, we investigated whether parent genes had known oncogenic or tumor suppressive roles. We performed a literature search to determine if any had been previously described in the context 
TABLE 2 | Parent genes of deregulated $\Psi$-Incs previously described in cancer literature.

\begin{tabular}{|c|c|c|c|c|c|c|c|}
\hline Parent Gene & Cancer $^{a}$ & Lung $^{\text {a }}$ Cancer & $\Psi$-Inc & Parent Gene & Cancer ${ }^{a}$ & Lung $^{\mathrm{a}}$ Cancer & $\Psi$-IncRNA \\
\hline$A D C$ & 8 & - & RP11-439L8.3 & MT1 & $>1000$ & 95 & MMP24-AS1 \\
\hline$A G A P 1$ & 12 & - & AGAP11 & $N E B L$ & 17 & - & LINC00342 \\
\hline$A R I H 1$ & 4 & - & $R P 11-1007024.3$ & NUP210 & 9 & 2 & H1FX-AS1 \\
\hline ATXN7L3 & 5 & - & $R P 11-56 G 10.2$ & PCBP2 & 26 & - & PCBP1-AS1 \\
\hline$B C R$ & $>1000$ & $>1000$ & AC008132.13 & $P K D 1$ & 238 & 5 & $R P 11-1186 N 24.5$ \\
\hline BMS1 & 4 & - & AGAP11 & POTEF & 4 & - & RP11-193Н5.1 \\
\hline CDC42 & 1657 & 129 & $R P 11-390 F 4.3$ & PPARGC1B & 37 & 1 & $R P 11-527 N 22.1$ \\
\hline \multirow[t]{2}{*}{ CECR7 } & 3 & - & AP000525.9; & $P P Y$ & 91 & 5 & CTD-2008P7.8 \\
\hline & & & CTD-2314B22.3 & PRKX & 12 & 2 & $R P 11-526 / 2.1$ \\
\hline CELSR1 & 11 & 2 & DGCR5 & PTCHD3 & 2 & - & PTCHDЗP1 \\
\hline CHRNB1 & 8 & - & RP11-650L12.2 & PTMA & 34 & - & LINC00987 \\
\hline $\mathrm{ClC}$ & - & 96 & $R P 11-34 P 13.7$ & $P Z P$ & 20 & - & LINC00987 \\
\hline \multirow[t]{2}{*}{ CS } & - & 10 & LINC00883; & $R A B 11 F I P 1$ & 16 & 1 & $R P 3-368 A 4.5$ \\
\hline & & & RP11-446H18.5 & $R A B 40 B$ & 5 & - & LLOXNC01-25OH12.3 \\
\hline CSPG4 & 62 & 5 & DNM1P51 & $R C N 1$ & 17 & 1 & TPT1-AS1 \\
\hline CTAGE1 & 49 & - & $R P 1-122 P 22.2$ & RNASEH1 & 7 & - & RP11-344E13.3 \\
\hline CYP4F2 & 29 & - & CYP4F35P & RPL21 & 5 & - & AC005062.2 \\
\hline CYP4F3 & 10 & 1 & FAM95B1 & RPL23A & 8 & - & $H L A-F-A S 1$ \\
\hline \multirow[t]{2}{*}{ CYP4F31P } & 10 & 1 & CYP4F35P; & $R P S A$ & 51 & 3 & $F T X$ \\
\hline & & & FAM95B1 & RPSAP58 & - & 3 & LINC00466 \\
\hline$D F F B$ & 33 & 1 & TOPORS-AS1 & SEMA3A & 163 & 25 & SEMA3B \\
\hline DRD2 & 121 & 10 & AP000438.2 & SHQ1 & 10 & 1 & RP11-50E11.3 \\
\hline EGLN1 & 158 & 5 & RP11-182J1.1 & SNAPC5 & 4 & - & LY86-AS1 \\
\hline FAM103A1 & 1 & - & $R P 11-324 H 6.5$ & SNX18 & 2 & - & $R P 11-435 B 5.5$ \\
\hline GPR39 & 8 & - & $R P 11-399019.9$ & SRSF9 & 5 & - & $R P 11-752 G 15.3$ \\
\hline \multirow[t]{2}{*}{ HMGB1 } & $>1000$ & 111 & RP11-349A22.5; & TACC3 & 122 & 21 & LINC00667 \\
\hline & & & ZBED3-AS1 & TOMM40 & 10 & 2 & CTD-2314B22.3 \\
\hline HMGN2P46 & 81 & 1 & RAMP2-AS1 & TPTE & 69 & 7 & TPTEP1 \\
\hline KRT8 & 94 & 7 & RP5-1198020.4 & TUBB4B & 5 & - & RP11-386G11.10 \\
\hline \multirow[t]{2}{*}{ LINC00657 } & 2 & - & CTA-14H9.5; & TULP3 & 5 & - & LINC00359 \\
\hline & & & HCG11 & VENTX & 8 & 1 & RP11-81H3.2 \\
\hline LMNB2 & 3 & 1 & RP11-161H23.9 & $W W F$ & 733 & 52 & TPTEP1 \\
\hline MARK4 & 25 & 3 & CTD-2201G3.1 & ZNF14 & 2 & - & CTD-2666L21.1; \\
\hline MICE & 1 & - & $H L A-F-A S 1$ & & & & ZNF833P \\
\hline \multirow[t]{2}{*}{ MIPEPP3 } & 4 & 1 & C1QTNF9B-AS1 & ZNF44 & 1 & - & CTD-2666L21.1 \\
\hline & & & & ZNF584 & 1 & - & CTD-2619J13.17 \\
\hline
\end{tabular}

a denotes number of entries in PubMed.

of cancer. Interestingly, we found that 65 of these parent genes had been previously described in cancer, and of those, 33 had been described in lung cancer (Table 2). Of the 34 significantly differentially expressed $\Psi$-lnc-parent gene pairs, 25 parent genes were described in cancer. This includes lung cancer associated genes like CS, which affects tumor drug response, as well as RCN1, which is associated with poor prognosis and tumor progression in lung cancer (Chen et al., 2014, 2018).

As the vast majority of the deregulated $\Psi$-lncs that were correlated with their parent gene had positive associations, we were interested whether this was a global phenomenon or exclusive to deregulated genes. We performed a Spearman's correlation analysis on every $\Psi$-lnc-parent-gene pair with expression data in our dataset irrespective of deregulation status ( $n=390$ gene pairs). We plotted the distribution of Spearman's rho $(\rho)$ values for the $\Psi$-lnc-parent-gene pairs and compared them to the rho values for $\Psi$-lncs paired to randomly selected genes. We found that the $\Psi$-lnc-parentgene pairs have significantly more positive relationships than the random gene pairs in both the BCCA (Mann Whitney $U$-test, $p<0.0001$ ) and TCGA datasets (Mann Whitney $U$-test, $p<0.0001$; Figure 4A). Studies have shown that IncRNAs transcribed from opposite strands can have different regulatory effects on target genes (Johnsson et al., 2013; Balbin et al., 2015). To determine if transcriptional orientation has an effect on $\Psi$-lnc-parent relationships we compared the Spearman's rho values of sense $\Psi$-lnc-parent pairs to antisense $\Psi$-lnc-parent pairs. In both datasets we observed that the sense $\Psi$-lncparent pairs to have significantly more positive relationships than the antisense $\Psi$-lnc-parent pairs (Mann Whitney $U$-test, TCGA set $(p<0.0001)$, and BCCA set $(p<0.0025)$ (Figure 4B and Supplementary Table S6). Strongly positively 
A

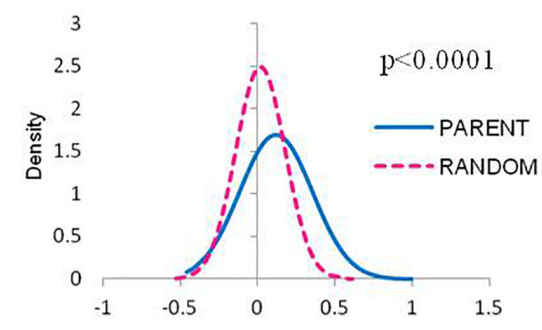

C

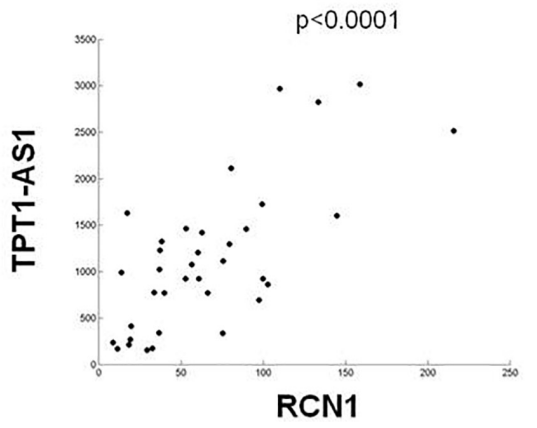

B
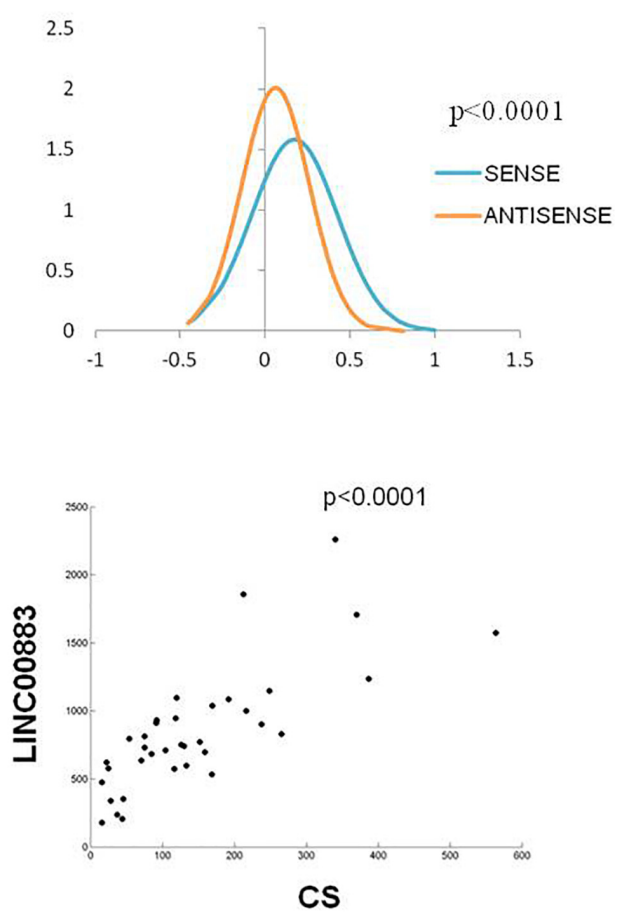

FIGURE 4 | Distribution of Spearman's Correlation rho values for all $\Psi$-Inc-parent-gene pairs in the TCGA dataset $(n=391)$. (A) Distribution of Spearman's correlation coefficients between $\Psi$-Inc-parent gene pairs (blue line, median $R=0.088$ ) and $\Psi$-Inc-random gene pairs (pink dashed line, median $R=0.019$ ). Rho values of the groups were compared by Mann Whitney U-test. (B) Distribution of Spearman's correlation coefficients between sense $\Psi$-Inc-parent-gene pairs (turquoise line, median $R=0.140$ ) and antisense $\Psi$-Inc-parent-gene pairs (orange line, median $R=0.049$ ). (C) Correlation scatter plots of $\Psi$-Incs with positive expression correlations to cancer-associated parent genes.

correlated $\Psi$-lnc-parent pairs include TPT1-AS1/RCN1 and LINC00887/CS (Figure 4C).

\section{$\Psi$-Incs and Their Parent Genes Are Associated With Patient Survival}

If the aberrant expression of $\Psi$-lncs is biologically relevant, it follows that they may be relevant in tumor aggressiveness, stage, and patient survival. We performed a two-group analysis using a Mann Whitney $U$-test between Stage I tumors and Stage II-IV tumors, as the majority of our tumors fell into these categories (Table 1). Of the deregulated $\Psi$-lncs we found CTC-250I14.3 to be associated with Stage 1 disease and downregulated in both the BCCA and TCGA LUAD cohorts (Supplementary Figure S1). Our discovery datasets were limited in sample size for survival association analysis; therefore, we examined a third cohort of 719 LUAD from the KM Plotter database (Kaplan-Meier Plotter) ${ }^{1}$ (Table 1). This cohort was limited to genes with probe coverage on microarray platforms. A total of 19 of the deregulated $\Psi$-lncs were represented on this platform, yet, the majority of these $\Psi$-lncs (16 of 19) were significantly associated with poor overall survival $(\log -\operatorname{rank} p<0.05$, Table 3$)$.

While we were not able to investigate the survival associations of all deregulated $\Psi$-lncs as many were not covered by the microarray platforms, the majority (72 out of 103) of

${ }^{1}$ http://kmplot.com/analysis/ their parent genes were represented. We discovered that 67 of these parent genes were associated with patient survival. Twenty-eight of these survival associated parent genes were also significantly associated with the expression of their paired deregulated $\Psi$-lnc. Furthermore, we found 11 pairs where both $\Psi$-lnc and parent gene are associated with patient survival. For example RP11-1007O24.3, a $\Psi$-lnc downregulated in tumors, is positively associated with expression of survival-associated parent gene ARIH1 in both the BCCA and TCGA datasets (Table 3 and Figure 5A). Further examples also include $\Psi$-lnc-parent pairs such as ZBED3-AS1 and HMGB1, which are positively correlated at the expression level, and both significantly associated with survival (Figure 5B). We also observe $\Psi$-lncparent pairs that are associated with survival, but do not share an expression relationship such as LINC00667 and parent gene TACC3 (Figure 5C). Collectively, our discovery of the broad deregulation of $\Psi$-lncs, many of which are survival-associated and associated with parent gene expression, may indicate that $\Psi$-lncs impact LUAD biology through trans regulation of their cancer-associated parent genes.

\section{DISCUSSION}

Here we expand upon the work done by Milligan et al. (2016), completing the first large-scale analysis of lncRNA expression from pseudogene loci in LUAD and paired non-malignant lung 
TABLE 3 | Associations between $\Psi$-IncRNA, parent gene expression, and patient outcome.

\begin{tabular}{|c|c|c|c|c|c|}
\hline$\Psi$-IncRNA & $\begin{array}{l}\text { Survival } p \\
\text { (KmPlotter) }\end{array}$ & $\begin{array}{l}\text { Parent } \\
\text { Gene }\end{array}$ & $\begin{array}{c}\text { Survival p } \\
\text { (KmPlotter) }\end{array}$ & $\begin{array}{c}\text { Association } \\
\text { with Parent } \\
(p, \mathrm{BCCA})\end{array}$ & $\begin{array}{c}\text { Association } \\
\text { with Parent } \\
\text { (p, TCGA) }\end{array}$ \\
\hline AC008132.13 & - & $B C R$ & $0.00043^{a}$ & 0.0173 & - \\
\hline AGAP11 & $0.0099^{b}$ & AGAP1 & $5.80 \mathrm{E}-10^{\mathrm{b}}$ & 0.0005 & - \\
\hline AGAP11 & - & $B M S 1$ & $0.00018^{a}$ & 0.0121 & - \\
\hline AP000525.9 & - & CECR7 & $3.20 \mathrm{E}-06^{\mathrm{a}}$ & - & 0.0371 \\
\hline CTA-14H9.5 & - & LINC00657 & $6.00 \mathrm{E}-06^{\mathrm{b}}$ & - & - \\
\hline CTD-2314B22.3 & - & CECR7 & $3.20 \mathrm{E}-06^{\mathrm{a}}$ & - & 0.0402 \\
\hline CTD-2619J13.17 & - & ZNF584 & $1.70 \mathrm{E}-08^{\mathrm{a}}$ & - & 0.0205 \\
\hline CTD-2666L21.1 & - & ZNF44 & $2.00 \mathrm{E}-15^{\mathrm{b}}$ & - & 0.0013 \\
\hline CTD-2666L21.1 & - & ZNF14 & $1.90 \mathrm{E}-12^{\mathrm{a}}$ & - & - \\
\hline DGCR5 & $4.00 \mathrm{E}-04^{\mathrm{a}}$ & CELSR1 & $2.70 \mathrm{E}-02^{\mathrm{a}}$ & - & - \\
\hline FAM66C & $3.00 \mathrm{E}-04^{\mathrm{b}}$ & DEFB130 & - & - & - \\
\hline FAM95B1 & - & CYP4F3 & 2.00E-01 ${ }^{\mathrm{b}}$ & - & - \\
\hline HCG11 & $1.40 \mathrm{E}-08^{\mathrm{b}}$ & LINC00657 & $6.00 \mathrm{E}-06^{\mathrm{b}}$ & - & 0.0046 \\
\hline LINC00342 & $1.80 \mathrm{E}-06^{\mathrm{b}}$ & $N E B L$ & $2.30 \mathrm{E}-05^{\mathrm{b}}$ & - & - \\
\hline LINC00466 & $4.60 \mathrm{E}-06^{\mathrm{a}}$ & RPSAP58 & - & - & - \\
\hline LINC00639 & $0.0087^{b}$ & ZFP41 & $0.014^{b}$ & - & - \\
\hline LINC00667 & $4.50 \mathrm{E}-08^{\mathrm{b}}$ & TACC3 & $6.20 \mathrm{E}-09^{\mathrm{a}}$ & - & - \\
\hline LINC00883 & $2.90 \mathrm{E}-03^{\mathrm{b}}$ & CS & $0.00041^{a}$ & 0.0449 & - \\
\hline LINC00957 & $0.042^{b}$ & RASA4B & $0.035^{a}$ & - & - \\
\hline LINC00982 & $1.30 \mathrm{E}-06^{\mathrm{b}}$ & $n / a^{c}$ & - & - & - \\
\hline LINC00987 & - & $P Z P$ & $0.02^{\mathrm{a}}$ & - & $<0.0001$ \\
\hline LY86-AS1 & $0.00025^{b}$ & SNAPC5 & $5 e-0.6^{b}$ & - & - \\
\hline MMP24-AS1 & $0.023^{a}$ & MT1 & $1.20 \mathrm{E}-12^{\mathrm{a}}$ & - & - \\
\hline PCBP1-AS1 & - & PCBP2 & 4. $30 \mathrm{E}-13^{\mathrm{b}}$ & - & - \\
\hline RAMP2-AS1 & $0.014^{b}$ & HMGN2P46 & - & - & - \\
\hline RP11-1007024.3 & - & $A R I H 1$ & $0.00093^{b}$ & $<0.0001$ & 0.0030 \\
\hline RP11-1186N24.5 & - & PKD1 & $0.0013^{b}$ & - & $<0.0001$ \\
\hline RP11-182J1.1 & - & EGLN1 & $1.20 \mathrm{E}-08^{\mathrm{b}}$ & 0.0011 & - \\
\hline$R P 11-344 E 13.3$ & - & RNASEH1 & $1.00 \mathrm{E}-07^{\mathrm{b}}$ & 0.0007 & - \\
\hline$R P 11-349 A 22.5$ & - & HMGB1 & $7.50 \mathrm{E}-10$ & - & - \\
\hline$R P 11-34 P 13.7$ & - & $\mathrm{ClC}$ & $1.20 \mathrm{E}-08^{\mathrm{a}}$ & - & 0.0205 \\
\hline RP11-390F4.3 & - & $C D C 42$ & 1.30E-05 & 0.0449 & - \\
\hline RP11-439L8.3 & - & $A D C$ & 3.30E-09 & 0.0205 & - \\
\hline RP11-446H18.5 & - & CS & $0.00041^{a}$ & - & - \\
\hline$R P 11-93 K 22.13$ & - & FAM86B3P & $6.30 \mathrm{E}-05^{\mathrm{b}}$ & - & 0.0129 \\
\hline TOPORS-AS1 & - & $D F F B$ & $9.10 \mathrm{E}-08^{\mathrm{b}}$ & - & 0.0033 \\
\hline TPT1-AS1 & $0.00019^{b}$ & RCN1 & $0.016^{a}$ & 0.0242 & - \\
\hline ZBED3-AS1 & 1.10E-10 & HMGB1 & $7.50 \mathrm{E}-10^{\mathrm{b}}$ & 0.0100 & - \\
\hline ZNF833P & - & ZNF491 & $0.016^{b}$ & - & - \\
\hline ZNF833P & - & ZNF14 & $1.90 \mathrm{E}-12^{\mathrm{a}}$ & - & 0.0079 \\
\hline
\end{tabular}

tissue. We discovered a broadly positive association between $\Psi$-lncs and parent-gene expression, suggestive of an alternative mechanism of cancer gene regulation. While there have been singular examples of deregulated pseudogene-derived lncRNAs in cancer, we show that this phenomenon is widespread in LUAD. In addition to being correlated with $\Psi$-lnc expression, we find that many of the parent genes of these deregulated lncRNAs are annotated cancer genes and are significantly associated with patient survival, highlighting how these previously unappreciated non-coding genes may affect LUAD biology.

While the identification of IncRNAs associated with cancer phenotypes is increasing, a great challenge in the field remains the accurate downstream prediction of lncRNA function. Unlike protein-coding genes or small non-coding RNAs, features like complex folding patterns and unknown binding motifs have contributed to the challenging functional characterization of lncRNAs. We utilized the sequence similarity found between lncRNAs expressed from pseudogene loci, and their parent genes to predict the function of this subset of lncRNAs in LUAD. We identified a set of $104 \Psi$-lncs deregulated in LUAD in two independent datasets. This greatly increases the number of deregulated lncRNAs known to be expressed from pseudogene loci in LUAD. Interestingly, the majority of these $\Psi$-lncs were under-expressed in tumors compared to non-malignant tissues, suggesting that they may have tumorsuppressive roles, and that their downregulation is advantageous to LUAD tumorigenesis.

Under-expression was not significantly associated with regions of recurrent copy number deletion in LUAD, although a subset of deregulated $\Psi$-lnc loci were localized to these regions (Figure 3). These observations suggest that they may be regulated by alternative molecular mechanisms, including broad chromosomal aberrations that affect whole chromosome arms, or epigenetic mechanisms. For example, endogenous retroviruses and repetitive elements often become aberrantly expressed in cancer due to deregulated methylation patterns (Kassiotis, 2014). We did not observe enrichment of $\Psi$-lncs or their parent genes on any chromosomes, despite the fact that pseudogenes are known to be overabundant on the human $\mathrm{X}$ chromosome (Drouin, 2006).

The direction of transcription often affects lncRNA function (Balbin et al., 2015). For example, the PTEN pseudogene (PTENP1) expressed in the sense direction can function as a decoy for inhibitory miRNAs that would otherwise cause translational inhibition of the PTEN parent mRNA. Conversely, when the antisense lncRNA is expressed from the PTENP1 locus, the transcript is able to localize to the PTEN parent locus and recruit chromatin-remodeling machinery, which leads to the silencing of PTEN transcription (Poliseno et al., 2010; Johnsson et al., 2013). Both mechanisms have been coopted by cancer cells for their respective tumor suppressive and oncogenic roles ( $\mathrm{Hu}$ et al., 2018). We found sense $\Psi$-lnc-parent pairs, (which account for 208 out of $391 \Psi$-lncs examined) to be more positively correlated than antisense $\Psi$-lnc-parent pairs in both cohorts (Figure 4B). This may imply that $\Psi$-lncs are more likely to regulate their parent gene in a positive manner which may occur through mechanisms such as miRNA sponging or transcript stabilization when transcribed in the sense direction (Glenfield and McLysaght, 2018; Hu et al., 2018). The distribution of Spearman's $\rho$-values for antisense-parent gene pairs suggests a more even split between positive and negative regulation. A limitation of this study is that we cannot discount the possibility that sequencing reads for sense overlapping $\Psi$-lncs that have sequence homology with their parent gene are being mapped to the parent gene instead of the $\Psi$-lnc. This potential issue warrants further investigation considering both the large number of annotated pseudogenes in the genome $(n=13,000)$ and the possibility of false interpretation of sequencing data for both protein-coding and non-coding genes. ${ }^{2}$ While these alignment errors could affect sense $\Psi$-lnc-parent gene pairs,

\footnotetext{
${ }^{2}$ https://www.genenames.org/cgi-bin/statistics
} 


\section{A}
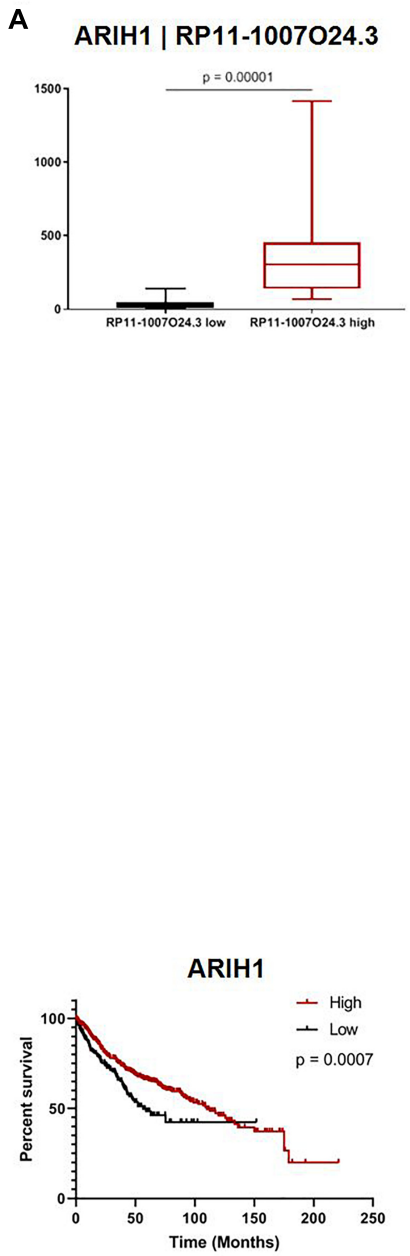

B

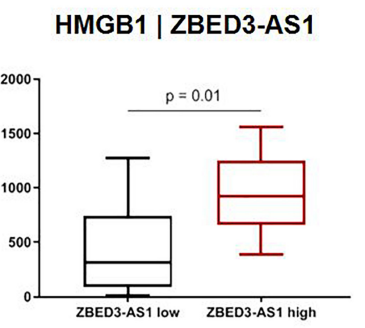

ZBED3-AS1

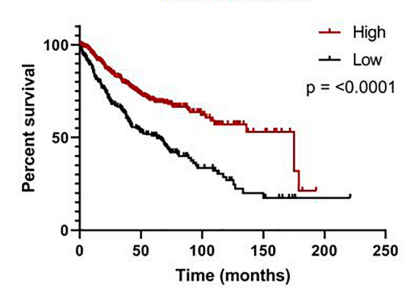

HMGB1

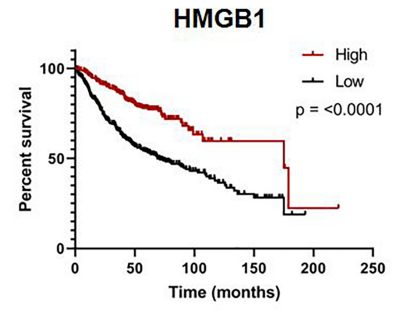

C

TACC3 | LINC00667

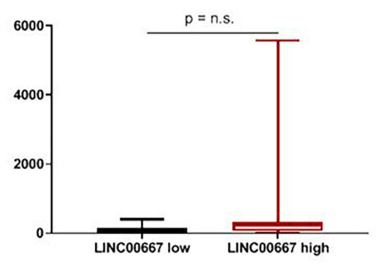

LINC00667

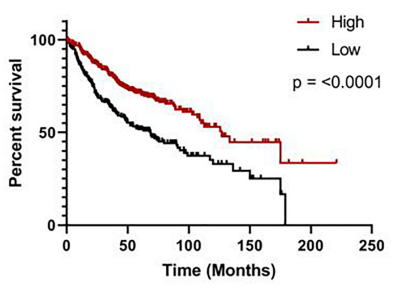

TACC3

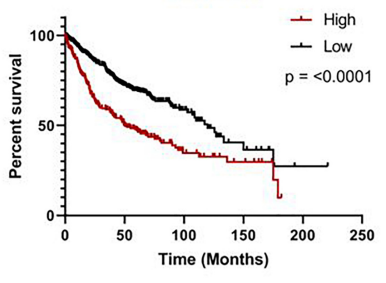

FIGURE 5 | Associations of pseudogene-derived IncRNAs (upper row), their respective parent gene expression levels and their potential impact on patient outcome (middle row and bottom row). Expression and survival associations as seen for: (A) RP11-1007O24.3 and parent gene ARIH1, (B) ZBED3-AS1 and parent gene HMGB1, and (C) LINC00667 and parent gene TACC3. Expression associations (upper row) for each pseudogene-derived IncRNA and parent gene pair were found by stratifying samples into tertiles by high (red) and low (black) expression of the pseudogene-derived IncRNA, and plotting the expression of the parent-gene (RPKM) on the $y$-axis. Survival associations were found for pseudogene-derived IncRNAs (middle row) and their respective parent genes (bottom row). Samples were stratified into tertiles with high (red) and low (black) expression of the gene-of-interest, and the significance of the associations were assessed using the logRank method through GraphPad Prism 8 software on data obtained from kmPlot $(n=673)$. Survival information was not available for RP11-1007O24.3 and it was thus omitted from this analysis.

antisense $\Psi$-lnc-parent gene pairs are not subjected to this same technical artifact. Recently, RNA-sequencing analysis strategies have emerged that begin to address this issue, and long read RNAsequencing could be used to reduce errors in sequence alignment.

When looking at the parent genes of these deregulated $\Psi$-lncs we were interested to find that many had previously described roles in cancer (Table 2). This includes EGLN1, a well described cancer gene involved in regulation of tumor hypoxia, and CDC42, an oncogene involved in cell cycle control (Chan and Giaccia, 2010; Maldonado and Dharmawardhane, 2018). Many of these deregulated $\Psi$-lncs were also associated with clinical parameters such as patient survival and patient stage, in addition to the correlated expression between $\Psi$-lncs and their parent genes (Table 3). For example, ZBED3-AS1 and $H M G B 1$ were positively correlated at the expression level, and low expression of both genes was associated with poor patient survival (Figure 5B). We also observed $\Psi$-lnc-parent pairs where both genes are associated with survival, but do not share an expression relationship. LINC00667 and parent gene TACC3, for example, are both survival-associated, but not correlated at the expression level. TACC3 is a component of the TACC3/ch-TOG/clathrin protein complex, and roles in complex assembly have been previously observed for lncRNAs (Munschauer et al., 2018; Figure 5C). Thus, it is possible that LINC00667 is involved in a form of regulation that would not affect transcript levels, including protein-complex assembly. While we were unable to assess survival associations for many of the deregulated $\Psi$-lncs, they may still impact patient survival 
through regulation of their parent genes. We found 28 out of 34 expression-associated $\Psi$-lnc-parent gene pairs to have parent genes associated with patient survival. RP11-1007024.3, for example, was positively correlated with survival-associated parent gene ARIH1 expression in both cohorts. ARIH1 has been previously described to be a mediator of DNA-damage response and mitophagy in cancer cells (Figure 5A; Perdomo et al., 1988; von Stechow et al., 2015). The potential regulatory impact of $\Psi$-lncs on their clinically relevant parent genes is considerable and may represent a novel avenue for targeted therapies. While this study focused on the broad effects of $\Psi$-lnc deregulation, future studies utilizing in vitro and in vivo experiments will be necessary to determine the specific mechanisms of parent gene regulation.

As $\Psi$-lncs may represent an unexplored area of cancerassociated parent gene regulation, their therapeutic relevance should be further explored. LncRNAs make ideal targets for therapies that target RNA products such as Antisense Oligonucleotide (ASO) therapies, since RNA is their final functional state, rather than the intermediate product for proteincoding genes (Crooke et al., 2018). In addition, ASOs are easier and less costly to develop than small molecule inhibitors, and are in development as aerosol sprays that may be ideal for lung cancer treatment (Kjems and Howard, 2012; Li and Chen, 2013). However, as ASOs target through complementary sequence pairing, they would have to be designed in such a way as to not interfere with the parent gene, especially in the case of $\Psi$-lncs expressed from the sense strand.

This strategy of identifying lncRNAs aberrantly expressed from pseudogene loci may be useful when applied to other cancer types. Indeed, we see that several of our deregulated $\Psi$-lncs have been described in other tumor types, such as TPT1AS1 in cervical cancer, and HGC11 in both prostate cancer and hepatocellular carcinoma (Zhang et al., 2016; Xu et al., 2017; Jiang et al., 2018). Additionally, as lncRNA expression is highly tissue specific, the application of this approach to other cancer types may yield novel disease-specific $\Psi$-lnc-parent pairs, highlighting the clinical utility of examining these previously underappreciated transcripts. Overall, $\Psi$-lnc-cancer-parent-gene axes represent alternative mechanisms of cancer gene regulation, and their identification is a critical step toward the functional characterization of lncRNAs.

\section{CONCLUSION}

There is a growing need to functionally characterize lncRNAs. Pseudogene-derived lncRNAs have been shown to be involved in cancer and regulate the expression of their parent genes. We show here how pervasive this gene regulatory mechanism is in LUAD samples. We identify a large set of deregulated $\Psi$-lncs, with aberrant expression observed in RNA-sequencing data from two LUAD cohorts of paired tumor and non-malignant lung tissue samples. We show that these deregulated $\Psi$-lncs have clinical value and that the parent genes, many of which are correlated with $\Psi$-lnc expression, have been implicated in cancer phenotypes and are associated with clinical outcome Together, our results highlight the important roles of the non-coding transcriptome in cancer cellular biology.

\section{MATERIALS AND METHODS}

\section{Next Generation Sequencing of Lung Adenocarcinoma Patient Samples Discovery Cohort}

We performed Next Generation Illumina HiSeq RNA sequencing on a set of 36 micro-dissected LUAD tumors and matched adjacent non-malignant tissue $(n=72)$. Our British Columbia Cancer Agency cohort (BCCA) was composed of fresh-frozen LUAD tumors and matched non-malignant lung parenchymal tissue collected from 36 patients at the Vancouver General Hospital with approval from the University of British ColumbiaBCCA Research Ethics Board. Consent obtained from the tissue donors of this study was both informed and written. To avoid field effects non-malignant samples were collected from areas $>2 \mathrm{~cm}$ away from the tumor. In order to reduce contaminating sequences derived from alternative cell types, tissue microdissection was guided by a pathologist. Samples used in this study contained $>80 \%$ tumor cell or $>80 \%$ nonmalignant cell content. Total RNA was extracted using Trizol reagent and standard procedures.

\section{Processing of RNA-Sequencing Data}

Total RNA was used for library construction at the Genome Sciences Center (GSC, Vancouver, BC, Canada). Briefly, samples were first analyzed using Agilent Bioanalyzer RNA nanochip, and samples that passed quality check were arrayed into a 96-well plate. PolyA+ RNA was purified using the 96-well MultiMACS mRNA isolation kit on the MultiMACS 96 separator (Miltenyi Biotec, Germany) from $2 \mu \mathrm{g}$ total RNA with on-column DNase I-treatment as per the manufacturer's instructions. Doublestranded cDNA was synthesized from the purified polyA ${ }^{+}$-RNA using the Superscript Double-Stranded cDNA Synthesis kit (Life Technologies, United States) and random hexamer primers at a concentration of $5 \mu \mathrm{M}$. The paired-end sequencing library was prepared following the GSC paired-end library preparation protocol, which is strand specific. Sequencing was performed using the Illumina HiSeq 2000 platform. Raw sequencing reads were subject to a quality control process. Reads with a length $<50 \mathrm{nt}$ (under two thirds of maximum read length of $75 \mathrm{nt}$ ) and quality level (Phred) $<20$ were discarded. High quality reads (.fastq files) were aligned to the NCBI GRCh37 reference human genome build using the STAR aligner (v 2.4.1d) under default parameters (Dobin et al., 2013). Aligned reads (.bam files) were quantified using Ensembl Transcripts (Release 75) reference annotations (Flicek et al., 2014). Raw RNA sequencing reads from each patient (tumor and corresponding non-malignant tissue) were deposited at BioProject. ${ }^{3}$ Quantification was performed using the Partek Flow platform as reads per kilobase per million (RPKM). RNA sequencing (.bam files) and clinical

\footnotetext{
${ }^{3}$ http://www.ncbi.nlm.nih.gov/bioproject/516232
} 
data for a secondary set of LUAD tumors and matched nonmalignant tissue $(n=108)$ were downloaded from The Cancer Genome Atlas (TCGA) Data Portal for validation purposes ${ }^{4}$ (Table 1). Expression profiles from TCGA were processed as described above.

\section{Identification of IncRNAs Expressed From Pseudogene Loci and Corresponding Parent Genes \\ $\Psi$-Inc Annotation}

Milligan et al. (2016) recently published a global atlas of lncRNAs that have exonic overlap with positionally nonredundant (unique) pseudogenes from 3 major pseudogene databases. Using this resource we obtained a list of lncRNAs overlapping pseudogene loci (Supplementary Table S1) that we used as a foundation for our expression analysis. As the degree of sequence overlap required for a pseudogenederived lncRNA to regulate its parent gene is unknown, we did not restrict our analysis to full-length, expressed pseudogenes, and included lncRNAs with any exonic overlap, including sense and antisense transcripts in order to annotate the most comprehensive list of $\Psi$-lncs (lncRNAs overlapping pseudogene loci).

\section{Parent Gene Annotation}

The parent gene information was also extracted for all pseudogenes overlapping our list of $\Psi$-lncs that have parentgene annotations in the YaleHuman60 and Retroali5 databases (Supplementary Table S7). Manual literature search was performed for parent genes of deregulated $\Psi$-lncs that were not contained in these databases.

\section{Statistical Analysis \\ Identification of Significantly Deregulated $\Psi$-Incs in Paired LUAD and Non-Malignant Lung Tissue}

Gene expression for protein-coding and non-coding genes was compared between tumors and non-malignant tissue and significantly deregulated genes were identified using a Wilcoxon signed-rank test $(p<0.05)$ and subjected to a BenjaminiHochberg $(\mathrm{BH})$ FDR correction. $\Psi$-lncs as identified previously were extracted, and those that were significantly deregulated between tumors and non-malignant tissue, in both our discovery (BCCA) and validation (TCGA) were selected for further analysis (Figure 1B).

\section{$\Psi$-Incs and Parent Gene Expression}

Tumors were sorted by $\Psi$-lnc expression for each $\Psi$-lncparent gene pair, and grouped into top and bottom $\Psi$-lnc expressing tertiles. Parent gene expression was then compared between the two groups using the Mann Whitney $U$-test ( $p$ value $\leq 0.05$ ). We performed a global expression analysis to determine whether $\Psi$-lnc-parent pairs were more positive or negatively correlated than random chance. For all $\Psi$-lncs with expression data in both datasets, Spearman's correlation rho

${ }^{4}$ https://portal.gdc.cancer.gov/ values were calculated for $\Psi$-lnc-parent gene pairs $(n=390)$ and compared to $\Psi$-lnc-random gene pairs. Random genes in our expression matrices were selected to pair with each $\Psi$-lnc. Each gene was assigned a number and pairs were chosen by using a random number generator. ${ }^{5}$ Spearman's rho values were then plotted, smoothed, and compared (Mann Whitney $U$-test, $p$-value $\leq 0.05)$. Rho value distribution was also compared between sense lnc-parent gene pairs $(n=208)$ and antisense lnc-parent pairs $(n=182)$.

\section{Literature Searches}

To determine if each gene of interest ( $\Psi$-lnc or parent gene) had been previously described in the context of tumors we searched Pubmed using the terms "gene + cancer" or "gene + lung cancer."

\section{Hierarchical Clustering and Data Visualization}

Unsupervised hierarchical clustering was performed in order to visualize and examine the expression of the $\Psi$-lncs in individual samples (Figure 2A). Average Linkage was used as a cluster distance metric, while Pearson Correlation was used as a point distance metric. To visualize the expression patterns of the most highly expressed $\Psi$-lncs, those with an average expression value of $\geq 10 \mathrm{RPKM}$ in either tumor or non-malignant samples were included in the analysis.

\section{Distribution of Deregulated $\Psi$-Incs Across Genome}

Locations of $\Psi$-lncs and parent genes were compared to identify their genomic position (Supplementary Table S4). Circular plot visualization was performed using the R-package Circlize (Figure 3; Gu et al., 2014). LUAD-specific regions of significant recurrent somatic copy number alterations had been previously identified by TCGA, and were used in this study to determine if the deregulated $\Psi$-lncs overlapped with frequently altered regions (Zack et al., 2013). All genomic coordinates correspond to the NCBI GRCh37 reference human genome build.

\section{Clinical Features}

\section{Survival Analysis}

A large public clinical database (Kaplan-Meier Plotter ${ }^{6}$ comprised of 719 LUAD samples was used to determine the association between both protein-coding and non-coding gene expression with patient outcomes. Similar to the BCCA and TCGA cohorts, the patient samples in this 3rd dataset were mostly comprised of Stage I and Stage II tumors (Table 1). Of the 104 deregulated $\Psi$-lncs, 19 were represented in this database, while $70 \%$ of parent genes (72 out of 103) were present. Default settings were used and a log-rank (Mantel-Cox) test was applied to compare survival between groups of tumors with high and low expression of each gene tested, where $p<0.05$ was considered statistically significant. The optimal expression cut-off was selected for each gene.

\footnotetext{
${ }^{5}$ https://www.random.org/

${ }^{6}$ http://kmplot.com/analysis/
} 


\section{Association With Tumor Stage}

The majority of tumors for BCCA and TCGA fell into the categories of Stage I and Stage II (Table 1). We compared expression of deregulated $\Psi$-lncs between Stage I tumors and tumors classified as Stage II and above using a Mann Whitney $U$-test $(p$-value $\leq 0.05)$.

\section{AUTHOR CONTRIBUTIONS}

GS was responsible for the project design. GS, KE, AS, VM, BM, MP, EM, and WL contributed to data acquisition, data analysis, interpretation of results, and manuscript preparation. WL was the principal investigator of this project. All authors have read, edited, and approved the final manuscript.

\section{FUNDING}

This study was supported by grants from the Canadian Institutes for Health Research (CIHR FDN-143345) and Scholarships from CIHR. EM is a Vanier Canada Graduate Scholar, and is additionally supported by UBC Faculty of Medicine.

\section{REFERENCES}

Arab, K., Park, Y. J., Lindroth, A. M., Schafer, A., Oakes, C., Weichenhan, D., et al. (2014). Long noncoding RNA TARID directs demethylation and activation of the tumor suppressor TCF21 via GADD45A. Mol. Cell 55, 604-614. doi: 10.1016/j.molcel.2014.06.031

Balbin, O. A., Malik, R., Dhanasekaran, S. M., Prensner, J. R., Cao, X., Wu, Y. M., et al. (2015). The landscape of antisense gene expression in human cancers. Genome Res. 25, 1068-1079. doi: 10.1101/gr.180596.114

Bhan, A., Soleimani, M., and Mandal, S. S. (2017). Long noncoding RNA and cancer: a new paradigm. Cancer Res. 77, 3965-3981. doi: 10.1158/0008-5472. CAN-16-2634

Blume, C. J., Hotz-Wagenblatt, A., Hullein, J., Sellner, L., Jethwa, A., Stolz, T., et al. (2015). p53-dependent non-coding RNA networks in chronic lymphocytic leukemia. Leukemia 29, 2015-2023. doi: 10.1038/leu. 2015.119

Chan, B. A., and Hughes, B. G. (2015). Targeted therapy for non-small cell lung cancer: current standards and the promise of the future. Transl. Lung. Cancer Res. 4, 36-54. doi: 10.3978/j.issn.2218-6751.2014.05.01

Chan, D. A., and Giaccia, A. J. (2010). PHD2 in tumour angiogenesis. Br. J. Cancer 103, 1-5. doi: 10.1038/sj.bjc.6605682

Chen, E. G., Zhang, J. S., Xu, S., Zhu, X. J., and Hu, H. H. (2017). Long non-coding RNA DGCR5 is involved in the regulation of proliferation, migration and invasion of lung cancer by targeting miR-1180. Am. J. Cancer Res. 7, 1463-1475.

Chen, L., Liu, T., Zhou, J., Wang, Y., Wang, X., Di, W., et al. (2014). Citrate synthase expression affects tumor phenotype and drug resistance in human ovarian carcinoma. PLoS One 9:e115708. doi: 10.1371/journal.pone.0115708

Chen, X., Shao, W., Huang, H., Feng, X., Yao, S., and Ke, H. (2018). Overexpression of RCN1 correlates with poor prognosis and progression in non-small cell lung cancer. Hum. Pathol. 83, 140-148. doi: 10.1016/j.humpath.2018.08.014

Crooke, S. T., Witztum, J. L., Bennett, C. F., and Baker, B. F. (2018). RNA-targeted therapeutics. Cell Metab. 27, 714-739. doi: 10.1016/j.cmet.2018.03.004

Dobin, A., Davis, C. A., Schlesinger, F., Drenkow, J., Zaleski, C., Jha, S., et al. (2013). STAR: ultrafast universal RNA-seq aligner. Bioinformatics 29, 15-21. doi: 10.1093/bioinformatics/bts635

Dong, H. X., Wang, R., Jin, X. Y., Zeng, J., and Pan, J. (2018). LncRNA DGCR5 promotes lung adenocarcinoma (LUAD) progression via inhibiting hsa-mir-22-3p. J. Cell Physiol. 233, 4126-4136. doi: 10.1002/jcp. 26215

\section{SUPPLEMENTARY MATERIAL}

The Supplementary Material for this article can be found online at: https://www.frontiersin.org/articles/10.3389/fgene. 2019.00138/full\#supplementary-material

FIGURE S1 | Expression of IncRNA CTC-250114.3 is significantly associated with Stage I disease in both the BCCA and TCGA LUAD cohorts (Mann Whitney U-test).

TABLE S1 | Detailed information of all pseudogene-derived IncRNAs.

TABLE S2 | Statistical analysis of the deregulated IncRNAs in the BCCA and TCGA cohorts (BHC-corrected $p$-values).

TABLE S3 | Number of PUBMED entries for IncRNA cancer-association.

TABLE S4 | Genomic locations of significant IncRNAs and their respective parent genes.

TABLE S5 | Expression correlations between the IncRNAs and their resective parent genes in the BCCA and TCGA cohorts.

TABLE S6 | Spearman's correlation coefficients for global expression analysis of $\psi$-Inc-parent pairs.

TABLE S7 | Parent gene information for pseudogenes contained in Retrolali5 and Yale60 databases.

Drouin, G. (2006). Processed pseudogenes are more abundant in human and mouse X chromosomes than in autosomes. Mol. Biol. Evol. 23, 1652-1655. doi: 10.1093/molbev/msl048

Feng, F., Qiu, B., Zang, R., Song, P., and Gao, S. (2017). Pseudogene PHBP1 promotes esophageal squamous cell carcinoma proliferation by increasing its cognate gene PHB expression. Oncotarget 8, 29091-29100. doi: 10.18632/ oncotarget.16196

Flicek, P., Amode, M. R., Barrell, D., Beal, K., Billis, K., Brent, S., et al. (2014). Ensembl 2014. Nucleic Acids Res. 42, D749-D755. doi: 10.1093/nar/gkt1196

Glenfield, C., and McLysaght, A. (2018). Pseudogenes provide evolutionary evidence for the competitive endogenous RNA hypothesis. Mol. Biol. Evol. 35, 2886-2899. doi: 10.1093/molbev/msy183

Gu, Z., Gu, L., Eils, R., Schlesner, M., and Brors, B. (2014). circlize Implements and enhances circular visualization in R. Bioinformatics 30, 2811-2812. doi: 10.1093/bioinformatics/btu393

Gutschner, T., Hammerle, M., Eissmann, M., Hsu, J., Kim, Y., Hung, G., et al. (2013). The noncoding RNA MALAT1 is a critical regulator of the metastasis phenotype of lung cancer cells. Cancer Res. 73, 1180-1189. doi: 10.1158/00085472.CAN-12-2850

Hirose, T., Virnicchi, G., Tanigawa, A., Naganuma, T., Li, R., Kimura, H., et al. (2014). NEAT1 long noncoding RNA regulates transcription via protein sequestration within subnuclear bodies. Mol. Biol. Cell 25, 169-183. doi: 10. 1091/mbc.E13-09-0558

$\mathrm{Hu}, \mathrm{X}$., Yang, L., and Mo, Y. Y. (2018). Role of pseudogenes in tumorigenesis. Cancers 10:256. doi: 10.3390/cancers10080256

Huang, J. L., Cao, S. W., Ou, Q. S., Yang, B., Zheng, S. H., Tang, J., et al. (2018). The long non-coding RNA PTTG3P promotes cell growth and metastasis via up-regulating PTTG1 and activating PI3K/AKT signaling in hepatocellular carcinoma. Mol. Cancer 17:93. doi: 10.1186/s12943-018-0841-x

Jiang, H., Huang, G., Zhao, N., Zhang, T., Jiang, M., He, Y., et al. (2018). Long non-coding RNA TPT1-AS1 promotes cell growth and metastasis in cervical cancer via acting AS a sponge for miR-324-5p. J. Exp. Clin. Cancer Res. 37:169. doi: 10.1186/s13046-018-0846-8

Johnsson, P., Ackley, A., Vidarsdottir, L., Lui, W. O., Corcoran, M., Grander, D., et al. (2013). A pseudogene long-noncoding-RNA network regulates PTEN transcription and translation in human cells. Nat. Struct. Mol. Biol. 20, 440-446. doi: $10.1038 / \mathrm{nsmb} .2516$

Kassiotis, G. (2014). Endogenous retroviruses and the development of cancer. J. Immunol. 192, 1343-1349. doi: 10.4049/jimmunol.1302972 
Khachane, A. N., and Harrison, P. M. (2009). Assessing the genomic evidence for conserved transcribed pseudogenes under selection. BMC Genomics 10:435. doi: 10.1186/1471-2164-10-435

Kjems, J., and Howard, K. A. (2012). Oligonucleotide delivery to the lung: waiting to inhale. Mol. Ther. Nucleic Acids 1:e1. doi: 10.1038/mtna.2011.1

Kung, J. T., Colognori, D., and Lee, J. T. (2013). Long noncoding RNAs: past, present, and future. Genetics 193, 651-669. doi: 10.1534/genetics.112. 146704

Lee, J. T., Davidow, L. S., and Warshawsky, D. (1999). Tsix, a gene antisense to Xist at the X-inactivation centre. Nat. Genet. 21, 400-404. doi: 10.1038/7734

Li, C. H., and Chen, Y. (2013). Targeting long non-coding RNAs in cancers: progress and prospects. Int. J. Biochem. Cell Biol. 45, 1895-1910. doi: 10.1016/j. biocel.2013.05.030

Luo, J., Zhu, H., Jiang, H., Cui, Y., Wang, M., Ni, X., et al. (2018). The effects of aberrant expression of LncRNA DGCR5/miR-873-5p/TUSC3 in lung cancer cell progression. Cancer Med. doi: 10.1002/cam4.1566 [Epub ahead of print].

Maldonado, M. D. M., and Dharmawardhane, S. (2018). Targeting Rac and Cdc42 GTPases in cancer. Cancer Res. 78, 3101-3111. doi: 10.1158/0008-5472.CAN18-0619

Milligan, M. J., Harvey, E., Yu, A., Morgan, A. L., Smith, D. L., Zhang, E., et al. (2016). Global intersection of long non-coding RNAs with processed and unprocessed pseudogenes in the human genome. Front. Genet. 7:26. doi: 10.3389/fgene.2016.00026

Munschauer, M., Nguyen, C. T., Sirokman, K., Hartigan, C. R., Hogstrom, L., Engreitz, J. M., et al. (2018). The NORAD lncRNA assembles a topoisomerase complex critical for genome stability. Nature 561, 132-136. doi: 10.1038/ s41586-018-0453-z

Peng, W. X., Koirala, P., and Mo, Y. Y. (2017). LncRNA-mediated regulation of cell signaling in cancer. Oncogene 36, 5661-5667. doi: 10.1038/onc.2017.184

Perdomo, R., Alvarez, C., Geninazzi, H., Ferreira, C., Monti, J., Parada, R., et al. (1988). Early diagnosis of hydatidosis by ultrasonography. Lancet 1:244. doi: 10.1016/S0140-6736(88)91097-5

Poliseno, L., Salmena, L., Zhang, J., Carver, B., Haveman, W. J., and Pandolfi, P. P. (2010). A coding-independent function of gene and pseudogene mRNAs regulates tumour biology. Nature 465, 1033-1038. doi: 10.1038/nature09144

Simon, M. D., Pinter, S. F., Fang, R., Sarma, K., Rutenberg-Schoenberg, M., Bowman, S. K., et al. (2013). High-resolution Xist binding maps reveal twostep spreading during X-chromosome inactivation. Nature 504, 465-469. doi: 10.1038 /nature 12719

Sun, M., Nie, F. Q., Zang, C., Wang, Y., Hou, J., Wei, C., et al. (2017). The pseudogene DUXAP8 promotes non-small-cell lung cancer cell proliferation and invasion by epigenetically silencing EGR1 and RHOB. Mol. Ther. 25, 739-751. doi: 10.1016/j.ymthe.2016.12.018
Tano, K., Mizuno, R., Okada, T., Rakwal, R., Shibato, J., Masuo, Y., et al. (2010). MALAT-1 enhances cell motility of lung adenocarcinoma cells by influencing the expression of motility-related genes. FEBS Lett. 584, 4575-4580. doi: 10. 1016/j.febslet.2010.10.008

von Stechow, L., Typas, D., Carreras Puigvert, J., Oort, L., Siddappa, R., Pines, A., et al. (2015). The E3 ubiquitin ligase ARIH1 protects against genotoxic stress by initiating a 4EHP-mediated mRNA translation arrest. Mol. Cell. Biol. 35, 1254-1268. doi: 10.1128/MCB.01152-14

Wang, K. C., Yang, Y. W., Liu, B., Sanyal, A., Corces-Zimmerman, R., Chen, Y., et al. (2011). A long noncoding RNA maintains active chromatin to coordinate homeotic gene expression. Nature 472, 120-124. doi: 10.1038/nature 09819

Xu, Y., Zheng, Y., Liu, H., and Li, T. (2017). Modulation of IGF2BP1 by long noncoding RNA HCG11 suppresses apoptosis of hepatocellular carcinoma cells via MAPK signaling transduction. Int. J. Oncol. 51, 791-800. doi: 10.3892/ijo.2017. 4066

Yamazaki, T., Fujikawa, C., Kubota, A., Takahashi, A., and Hirose, T. (2018). CRISPRa-mediated NEAT1 lncRNA upregulation induces formation of intact paraspeckles. Biochem. Biophys. Res. Commun. 504, 218-224. doi: 10.1016/j. bbrc.2018.08.158

Zack, T. I., Schumacher, S. E., Carter, S. L., Cherniack, A. D., Saksena, G., Tabak, B., et al. (2013). Pan-cancer patterns of somatic copy number alteration. Nat. Genet. 45, 1134-1140. doi: 10.1038/ng.2760

Zhang, M., Weng, W., Zhang, Q., Wu, Y., Ni, S., Tan, C., et al. (2018). The lncRNA NEAT1 activates Wnt/beta-catenin signaling and promotes colorectal cancer progression via interacting with DDX5. J. Hematol. Oncol. 11:113. doi: 10.1186/s13045-018-0656-7

Zhang, Y., Zhang, P., Wan, X., Su, X., Kong, Z., Zhai, Q., et al. (2016). Downregulation of long non-coding RNA HCG11 predicts a poor prognosis in prostate cancer. Biomed. Pharmacother. 83, 936-941. doi: 10.1016/j.biopha. 2016.08.013

Conflict of Interest Statement: The authors declare that the research was conducted in the absence of any commercial or financial relationships that could be construed as a potential conflict of interest.

Copyright (c) 2019 Stewart, Enfield, Sage, Martinez, Minatel, Pewarchuk, Marshall and Lam. This is an open-access article distributed under the terms of the Creative Commons Attribution License (CC BY). The use, distribution or reproduction in other forums is permitted, provided the original author(s) and the copyright owner(s) are credited and that the original publication in this journal is cited, in accordance with accepted academic practice. No use, distribution or reproduction is permitted which does not comply with these terms. 